\title{
Charles Miller Fisher, un grande de la neurología
}

\author{
JORGE TAPIA
}

Departamento de Neurología, Facultad de Medicina, Pontificia Universidad Católica de Chile. Santiago, Chile.

El autor declara no tener conflictos de intereses en este manuscrito.

Recibido el 24 de abril de 2013, aceptado el 31 de mayo de 2013

Correspondencia a: Dr. Jorge Tapia Marcoleta 350, piso 2. Santiago Centro. Teléfono: 56-2-23543316. E-mail: jftapia@med.puc.cl

\section{Charles Miller Fisher: A giant of neurology}

C. Miller Fisher MD, one of the great neurologists in the 20th century, died in April 2012. Born in Canada, he studied medicine at the University of Toronto. As a Canadian Navy medical doctor he participated in World War II and was a war prisoner from 1941 to 1944. He did a residency in neurology at the Montreal Neurological Institute between 1946 and 1948, and later on was a Fellow in Neurology and Neuropathology at the Boston City Hospital. In 1954 he entered the Massachusetts General Hospital as a neurologist and neuropathologist, where he remained until his retirement, in 2005. His academic career ended as Professor Emeritus at Harvard University. His area of special interest in neurology was cerebrovascular disease (CVD). In 1954 he created the first Vascular Neurology service in the world and trained many leading neurologists on this field. His scientific contributions are present in more than 250 publications, as journal articles and book chapters. Many of his articles, certainly not restricted to CVD, were seminal in neurology. Several concepts and terms that he coined are currently used in daily clinical practice. The chapters on CVD, in seven consecutive editions of Harrison's Internal Medicine textbook, are among his highlights. His death was deeply felt by the neurological community.

(Rev Med Chile 2013; 141: 1076-1080).

Key words: Cerebrovascular disorders; History of Medicine; Neurology.
$\mathrm{E}$ 114 de abril de 2012 murió Charles Miller Fisher, uno de los grandes neurólogos del siglo XX y, sin duda, el más importante neurólogo vascular (Figura 1).

Tuve la gran suerte de conocerlo y trabajar con él en los años 1981 y 1982, y posteriormente en 1992. Era una persona amable, acogedora, muy interesado en los demás, siempre tenía tiempo para ver pacientes o conversar de los más diferentes tópicos, como si el tiempo nada importara (muestra de ello era que no usaba reloj). Era muy meticuloso y preocupado en los detalles de la historia y examen clínico de los pacientes. No respondía directamente las preguntas que uno le hacía, sino que se daba el tiempo para contra preguntar por qué la hacía y qué pensaba uno al respecto.

El Dr. Fisher nació el 8 de diciembre de 1913 en Waterloo, Ontario, Canadá. Estudió medicina en la Universidad de Toronto; se tituló como Bachelor of Arts (BA) en 1935 y como Medical Doctor (MD) en 1938, realizando su internado entre 1938 y 1939 en el Hospital Henry Ford de Detroit, EEUU.

En septiembre de 1939 Canadá entró como aliado a la 2० Guerra Mundial, y en 1940 el Dr. Fisher ingresó como Surgeon Lieutenant a la Royal Canadian Navy. En 1941 fue destinado como médico al buque mercante inglés armado HMS Voltaire, que fue hundido el 4 de abril de 1941 en el Pacífico sur, dos días después que naciera su primera hija, Elizabeth. Luego de permanecer flotando en el mar durante $6 \mathrm{~h}$, fue rescatado por marinos alemanes y hecho prisionero de guerra. $\mathrm{La}$ mayor parte del tiempo permaneció en el campo Stalag XB en Sandbostel. Dada su gran inquietud intelectual, durante este período aprovechó de aprender alemán, italiano y algo de español, además de mecánica, navegación, matemáticas y teoría de la música. El 22 de junio de 1944 fue liberado, como médico acompañante de un grupo de pri- 
sioneros ingleses heridos que se intercambiaron por alemanes prisioneros de los aliados.

Para reinsertarse en la medicina, en 1945 inició una estadía de perfeccionamiento en el Royal Victoria Hospital de Montreal, con la intención de especializarse en diabetes y nutrición. Esta estadía incluyó una rotación de 6 semanas en el Montreal Neurological Institute, dirigido por el famoso neurocirujano Wilder Penfield. Durante este período recibieron un paciente que presentaba crisis epilépticas focales iniciadas con un ruido como un tambor. Estudiando el caso en la biblioteca, el Dr. Fisher encontró que a la circunvolución de Heschl llegaban los estímulos sonoros, y al día siguiente, durante la visita, el Dr. Penfield le preguntó qué creía él que tenía el paciente. El Dr. Fisher le respondió que pensaba que tenía un tumor en dicha estructura, lo que fue confirmado durante la cirugía. Este hecho, más las cualidades que encontró el Dr. Penfield en el Dr. Fisher, hicieron que éste lo convenciera de dedicarse a la neurología. Realizó una residencia en esta especialidad en el Montreal Neurological Institute entre 1946 y 1948. Una vez finalizada esta formación, el Dr. Penfield le planteó que estudiara los efectos de la hipertensión arterial en el encéfalo. Con este fin fue al Boston City Hospital, como Fellow en neurología bajo la dirección del Dr. Dereeck Denny-Brown, y neuropatología en el Mallory Institute, bajo la dirección del Dr. Raymond Adams.

Uno de los hechos que más lo impactó durante ésta estadía, fue el que ocurrió un día que tuvo que estudiar 9 encéfalos, encontrando en 3 de ellos un infarto hemorrágico en el territorio de la arteria cerebral media, y al revisar meticulosamente los vasos no encontró una oclusión arterial. Este era un hecho que contradecía la creencia, hasta ese momento, de que todos los infartos se debían a una trombosis arterial. Los 3 casos presentaban en vida una fibrilación auricular, lo que lo llevó a plantear que el mecanismo era una embolia, que luego se disgregaba recanalizándose espontáneamente el vaso. Esto motivó una de sus primeras publicaciones, el año 1951 junto con el Dr. Adams ${ }^{1}$.

Después de su formación en Boston, volvió al Montreal General Hospital, donde formó un Servicio de Neuropatología, y al Queen Victoria Hospital, donde atendió pacientes neurológicos. En este período y con pocas semanas de separación, le tocó atender a 2 pacientes que presentaron una hemiplejia y que referían que unas semanas antes

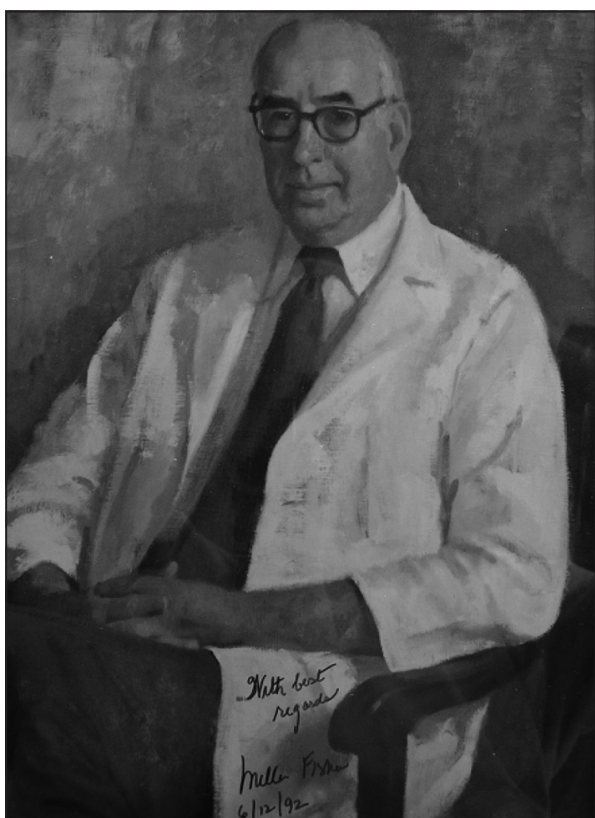

Figura 1. Fotografía cuadro Dr. C. M. Fisher del Servicio de Neurología del Massachusetts General Hospital, Boston.

habían sufrido una ceguera transitoria del ojo contralateral. Uno de ellos falleció meses más tarde de un cáncer de recto y el Dr. Fisher dirigidamente estudió sus carótidas, encontrando una oclusión aterotrombótica. A partir de este hecho interrogó sistemáticamente a los pacientes que presentaban accidentes vasculares encefálicos (AVE), refiriendo muchos de ellos síntomas premonitores a éstos, para los cuales el Dr. Fisher acuñó el término de "transient ischemic attack" (TIA)2 ${ }^{2}$ lo que motivó que en 1952 le dieran el Premio de Medicina en la reunión anual del Royal College of Physicians and Surgeons de Canadá. Preocupado por la prevención del AVE, planteó que en aquellos casos de TIA de origen carotideo, pudiera realizarse una cirugía de "by-pass" entre la carótida externa e interna. Estando de visita el Dr. Carrea, neurocirujano argentino, éste se interesó por la proposición del Dr. Fisher, y fue así como en octubre de 1951 hizo la primera cirugía de carótida en el mundo.

En 1953, Raymond Adams fue nombrado Bullard Professor de Neuropatología de la Universidad de Harvard y Jefe de Neurología del Massachusetts General Hospital (MGH), e invitó al Dr. Fisher a unirse a su grupo. Fue así como desde 1954 hasta 
su retiro en el 2005, fue neurólogo y neuropatólogo del MGH, y realizó una carrera académica de Profesor Asistente 1954-1964, Asociado 1964 1969, Titular 1969-1983 para luego ser nombrado Profesor Emérito de Neurología de la Escuela de Medicina de la Universidad de Harvard.

El Dr. Adams le encomendó continuar con el estudio de los AVE. Con este propósito creó en 1954 el primer servicio de neurología vascular del mundo, llegando rápidamente a atender a más de 100 pacientes al mes y teniendo como su principal base el Servicio de Urgencia. El Dr. Fisher decía que la neurología se aprendía "AVE tras AVE", y fueron muchos los neurólogos que se formaron en neurología vascular con él, destacando el Dr. J. Mohr, quien lo sucedió como jefe de este Servicio en 1973, y otros grandes neurólogos vasculares como Louis Caplan, Carlos Kase, Phillip Wolf, Michael Pessin, etc. Junto con el Dr. Adams y el Dr. E. Pierson Richardson, destacado neuropatólogo y neurólogo, constituyeron un equipo de excepción, formando muchas generaciones de neurólogos y publicando trabajos pioneros en neurología, que en el caso del Dr. Fisher suman más de 250. Durante los primeros años en Boston, su interés se centró principalmente en 3 puntos: 1) Uso de anticoagulantes en el AVE isquémico, participando probablemente en el primer estudio aleatorizado, multicéntrico, sobre el tratamiento anticoagulante en el infarto encefálico ${ }^{4}$. En esta línea, en 1972 planteó utilizar anticoagulantes para la prevención primaria de la embolia encefálica en la fibrilación auricular, lo que motivó un rechazo total de los cardiólogos de la época ${ }^{5}$. Su proposición se adelantó en 20 años a los trabajos que establecieron la conducta actual de anticoagulación de la fibrilación auricular no valvular; 2) Caracterización de los TIA, publicando en el año 1959 los hallazgos en el fondo de ojo de un paciente que presentaba una amaurosis transitoria secundaria a una estenosis carotidea, y en el cual durante una hora siguió el desplazamiento de un émbolo en las arterias retinianas, registrando simultáneamente la mejoría gradual del campo visual $^{6}$; 3) Caracterización y tratamiento quirúrgico de la hemorragia encefálica intraparenquimatosa hipertensiva. Hizo la distinción clínica entre la hemorragia putaminal y la talámica, que a la fecha se agrupaban como hemorragias ganglionares basales ${ }^{7}$. Describió las características clínicas de la hemorragia cerebelosa, permitiendo intervenir quirúrgicamente de modo precoz a estos pacientes en la era previa a la tomografía computarizada ${ }^{8}$. También describió el cuadro clínico, imitando una hemorragia subaracnoidea, de la hemorragia de la cabeza del caudado ${ }^{7}$. Este ordenamiento y caracterización de la clínica de las hemorragias, así como el de los principales síndromes neurológicos asociados a los infartos por oclusión de cada una de las grandes arterias encefálicas ${ }^{9}$, le permitieron escribir en forma excepcionalmente clara y docente el capítulo de enfermedades cerebrovasculares del libro de Medicina Interna de Harrison, desde su tercera edición en 1958, hasta su novena edición en $1980^{10}$.

El cuidado de los pacientes con AVE, le permitió evaluar en detalle a los enfermos con compromiso de conciencia, y así creó y presentó en 1959 una metodología de examen clínico que no existía en ese tiempo, para establecer la localización del daño que provocaba esta condición y su severidad, y que es la que se utiliza hasta la fecha ${ }^{11}$.

En 1967 publicó uno de los trabajos más importante en neurología, en el que describió diversas alteraciones neuro oftalmológicas, como la preservación del reflejo pupilar en la miosis por lesión pontina, la desviación conjugada de la mirada en el sentido incorrecto en la hemorragia talámica, el síndrome de $1 \frac{1}{2} 2$ en casos de lesiones pontinas, el valor localizador de los escotomas centellantes y del nistagmo vertical, el "bobbing" ocular en lesiones pontinas, y varios otros más que constituyen hasta la fecha pilares de la semiología neurológica $^{12,13}$.

Sin duda uno de los mayores aportes del Dr. Fisher en el campo de los AVE fue el de los infartos lacunares. A pesar de que fueron descritos por Dechambre en 1838, y Pierre Marie en 1901 planteó que se debían a una obstrucción arterial, fue el Dr. Fisher quien revivió este tema, y mediante estudios con cortes seriados estableció que en su mayoría se debían a la oclusión de una sola arteria penetrante, por una lesión in situ de su pared (lipohialinosis o por microateromas), estableciendo el concepto de que los infartos lacunares son un subtipo de AVE, con una forma propia de estudio, tratamiento y pronóstico, y describió los diferentes cuadros clínicos característicos de ellos. Sólo después de sus publicaciones, en las diferentes series clínicas de AVE aparecieron los infartos lacunares como un subtipo de AVE isquémico ${ }^{14-19}$.

Tuve el privilegio de estudiar con él el encéfalo de una paciente que había presentado un infarto 
que comprometía el bulbo, dando un síndrome de Wallenberg, y de puente, con el cuadro de infarto del territorio de la arteria cerebelosa antero inferior. Examinando dos mil preparaciones histológicas de cortes seriados, establecimos que se debía a la oclusión del ostium de origen de una rama de la arteria cerebelosa postero inferior, que irrigaba tanto el bulbo como la protuberancia ${ }^{20}$. El Dr. Fisher acuñó términos como el "robo de la subclavia" ${ }^{21}$ y hemiplejia "heráldica"22, este último para referirse a los episodios transitorios de hemiparesia que preceden a una oclusión de la basilar, los que se utilizan actualmente a diario en la clínica. También describió y estableció el término "signo de la cuerda", para la imagen angiográfica característica de la disección de la arteria carótida interna ${ }^{23}$. También describió las características del vértigo secundario a enfermedades cerebrovasculares $^{24}$, así como la cefalea asociada a éstos y su localización de acuerdo al territorio arterial comprometido $^{25}$. En 1980, describió la relación entre vasoespasmo y magnitud de la hemorragia subaracnoidea visualizada mediante la tomografía computarizada, creándose una escala que lleva su nombre y que se utiliza mundialmente ${ }^{26}$. En una publicación en que apareció él como último autor, acuñó el término y estableció como una entidad clínica el síndrome de vasoconstricción arterial segmentaria reversible, del que se habían publicado series pequeñas, y se le conoce como síndrome de Call-Fleming, que son los 2 primeros autores de esta publicación ${ }^{27}$.

El Dr. Fisher a su vez hizo importantes aportes en otros campos de la neurología. En el año 1956 describió una variante del síndrome de Guillain Barré, que se pasó a llamar Síndrome de Miller Fisher $^{28}$. Junto al Dr. Adams describió en 1958 la Amnesia Global Transitoria ${ }^{29}$. También fue él quien describió los equivalentes migrañosos que se pueden presentar en la edad adulta, como episodios de alteración neurológica transitorios, no asociados a cefalea, y las características que permiten diferenciarlos de un TIA y una crisis epiléptica $^{30}$. Asimismo, hizo importantes aportes en la descripción del cuadro clínico del Jakob Creutzfeldt, en especial a la alta frecuencia de la "reacción de sobresalto"31.

El Dr. Fisher se mantuvo muy activo durante los últimos años de su vida, y en 2003 publicó un caso en que, mediante cortes seriados, identificó por primera vez el sitio de rotura arterial de una hemorragia hipertensiva, a partir de un aneurisma de Charcot-Bouchard, que habían sido descritos en el siglo XIX ${ }^{32}$.

Su muerte ha sido muy sentida por la comunidad neurológica y se han publicado 4 obituarios en las principales revistas neurológicas.

\section{Referencias}

1. Fisher CM, Adams RD. Observations on brain embolism. J Neuropathol Exp Neurol 1951; 10: 92.

2. Fisher CM. Transient monocular blindness associated with hemiplegia. Trans Am Neurol Assoc 1951; 76: 1548.

3. Fisher CM. Disease of carotid arteries -a clinic- pathologic correlation. Report of Annual Meeting and Proceedings of the Royal College of Physicians and Surgeons of Canada, October 3-4, 1952, 60-7.

4. Baker RN, Broward JA, Fang HC, Fisher CM, Groch $\mathrm{SN}$, Heyman A, et al. Anticoagulant therapy in cerebral infarction. Neurology 1962; 12: 823-35.

5. Fisher CM. Treatment of chronic atrial fibrillation. Lancet 1972; 1: 1284. (letter to editor).

6. Fisher CM. Observations of the fundus oculi in transient monocular blindness. Neurology 1959; 9: 337-47.

7. Fisher CM. Clinical syndromes in cerebral hemorrhage. En: Fields W S, Editor, Pathogenesis and treatment of cerebrovascular disease. Springfield, USA: Editorial Charles C. Thomas; 1961, p. 318-38.

8. Fisher CM, Picard EH, Polak A, Dalal P, Ojemann RG. Acute hypertensive cerebellar hemorrhage: Diagnosis and surgical treatment. J Nerv Ment Dis 1965; 140: 3857.

9. Fisher CM. Clinical syndromes in cerebral arterial occlusion. En: Fields W S, Editor, Pathogenesis and treatment of cerebrovascular disease. Springfield, USA: Editorial Charles C. Thomas: 1961.p. 151-77.

10. Fisher CM, Karp HR, Adams RD. Cerebrovascular diseases. En: Harrison T R. et al, Editores, Principles of Internal Medicine. New York, USA: Editorial Mc GrawHill; 1958. p. 1560-606.

11. Fisher CM. The neurological examination of the comatose patient. Acta Neurol Scandinav 1969; (36) 45: 5-56.

12. Fisher CM. Some neuro-ophthalmological observations. J Neurol Neurosurg Psychiatry 1967; 30: 383-92.

13. Fisher CM. Ocular bobbing. Arch Neurol 1964; 11: 5436.

14. Fisher CM, Curry HB. Pure motor hemiplegia. Trans Am Neurol Assoc 1964; 89: 94-7.

15. Fisher C M. Pure sensory stroke involving face, arm, and 
leg. Neurology 1965; 15: 76-80.

16. Fisher CM, Cole M. Homolateral ataxia and crural paresis: A vascular syndrome. J Neurol Neurosurg Psychiatry 1965; 28: 45-55.

17. Fisher CM. Lacunares: Small, deep cerebral infarcts. Neurology 1965; 15: 774-84.

18. Fisher CM. A lacunar stroke. The dysarthria-clumsy hand syndrome. Neurology 1967; 17: 614-7.

19. Fisher CM. The arterial lesions underlying lacunes. Acta Neuropath 1969; 12: 1-15.

20. Fisher CM, Tapia J. Lateral medullary infarction extending to the lower pons. J Neurol Neurosurg Psychiatry 1987; 50: 620-4.

21. Fisher CM. A new vascular syndrome-"The subclavian steal”. N Engl J Med. 1961; 265: 912-3.

22. Fisher CM. The "Herald Hemiparesis" of basilar artery occlusion. Arch Neurol 1988; 45: 1301-3.

23. Ojemann RG, Fisher CM, Rich JC. Spontaneous dissecting aneurysm of the internal carotid artery. Stroke 1972; 3: 434-40.

24. Fisher CM. Vertigo in cerebrovascular disease. Arch Otolaryng 1967; 85: 529-34.

25. Fisher CM. Headache in cerebrovascular disease. En:
Vinken PJ, Bruyn GW, Editores, Handbook of clinical Neurology. Amsterdan, The Netherlands: Editorial North-Holland Publishing Company; 1967. p. 124-56.

26. Fisher CM, Kistler JP, Davis JM. Relation of cerebral vasospasm to subarachnoid hemorrhage visualized by computerized tomographic scanning. Neurosurgery 1980; 6: 1-9.

27. Call GK, Fleming MC, Sealfon S, Levine H, Kistler JP, Fisher CM. Reversible cerebral segmental vasoconstriction. Stroke 1988; 19: 1159-70.

28. Fisher CM. An unusual variant of acute idiopathic polyneuritis (syndrome of ophthalmoplegia, ataxia and areflexia). N Engl J Med 1956; 255: 57-65.

29. Fisher CM, Adams RD. Transient global amnesia. Trans Am Neurol Assoc 1958; 83: 146.

30. Fisher CM. Late-Life migraine accompaniments as a cause of unexplained transient ischemic Attacks. Can J Neurol Sci 1980; 7: 9-17.

31. Fisher CM. The clinical picture in Creutzfeldt-Jakob disease. Trans Am Neurol Assoc 1960; 85: 147-50.

32. Fisher CM. Hypertensive cerebral hemorrhage. Demostration of the source of bleeding. J Neuropathol Exp Neurol 2003; 62: 104-7. 